\title{
Microstructural alterations of sputum in cystic fibrosis lung disease
}

\author{
Gregg A. Duncan, ${ }^{1,2}$ James Jung, ${ }^{1,2}$ Andrea Joseph, ${ }^{1,3}$ Abigail L. Thaxton, ${ }^{4}$ Natalie E. West, ${ }^{4}$ \\ Michael P. Boyle, ${ }^{4,5}$ Justin Hanes, ${ }^{1,2,3,6}$ and Jung Soo Suk ${ }^{1,2}$ \\ ${ }^{1}$ Center for Nanomedicine and 2Department of Ophthalmology, Wilmer Eye Institute, Johns Hopkins University School of \\ Medicine, Baltimore, Maryland, USA. ${ }^{3}$ Department of Chemical and Biomolecular Engineering, Johns Hopkins University, \\ Baltimore, Maryland, USA. ${ }^{4}$ Division of Pulmonary and Critical Care Medicine, Department of Medicine, Johns Hopkins \\ University School of Medicine, Baltimore, Maryland, USA. ${ }^{5}$ Cystic Fibrosis Foundation, Bethesda, Maryland, USA. \\ ${ }^{6}$ Departments of Biomedical Engineering, Environmental and Health Sciences, Oncology, Neurosurgery, and Pharmacology \\ and Molecular Sciences, Johns Hopkins University, Baltimore, Maryland, USA.
}

The stasis of mucus secretions in the lungs of cystic fibrosis (CF) patients leads to recurrent infections and pulmonary exacerbations, resulting in decreased survival. Prior studies have assessed the biochemical and biophysical features of airway mucus in individuals with CF. However, these measurements are unable to probe mucus structure on microscopic length scales relevant to key players in the progression of CF-related lung disease, namely, viruses, bacteria, and neutrophils. In this study, we quantitatively determined sputum microstructure based on the diffusion of muco-inert nanoparticle probes in CF sputum and found that a reduction in sputum mesh pore size is characteristic of CF patients with reduced lung function, as indicated by measured FEV. We also discovered that the effect of ex vivo treatment of CF sputum with rhDNase I (Pulmozyme) on microstructure is dependent upon the time interval between the most recent inhaled rhDNase I treatment and the sample collection. Microstructure of mucus may serve as a marker for the extent of CF lung disease and as a parameter for assessing the effectiveness of mucus-altering agents.

Conflict of interest: The mucuspenetrating particle technology described in this publication is being developed by Kala Pharmaceuticals. J. Hanes is a cofounder of Kala Pharmaceuticals. He owns company stock, which is subject to certain restrictions under Johns Hopkins University policy. The terms of this arrangement are being managed by Johns Hopkins University in accordance with its conflict-of-interest policies.

Submitted: May 10, 2016 Accepted: September 29, 2016 Published: November 3, 2016

Reference information: JCI Insight. 2016;1(18):e88198. doi:10.1172/jii.insight.88198.

\section{Introduction}

Cystic fibrosis (CF) is one of the most common genetic disorders that primarily causes life-threatening lung disease (1). Recurrent pulmonary infections and acute exacerbations are the major causes of steady decline in lung function and shorter survival in CF (2-5). As the result of a defect in cystic fibrosis transmembrane conductance regulator (CFTR) proteins, homeostatic balance of sodium, chloride, and bicarbonate ions at the CF airway epithelial surface is misregulated, leading to dehydration of the airway surface liquid and increased concentration of mucus lining the airway $(6,7)$. The resulting increased viscosity of mucus leads to the inability to clear secretions, which causes chronic lung damage. DNA and other biomacromolecules released from necrotic neutrophils further concentrate airway secretions to form highly viscoelastic sputum (8). Recent studies in CF pig-based animal models have identified additional CFTR-mediated defects responsible for biochemical alterations of airway mucus, such as impairments in mucus detachment from submucosal glands and in $\mathrm{pH}$-dependent bacterial killing $(9,10)$. These pathophysiological changes render the airway mucus unable to be cleared efficiently by cough and mucociliary clearance (MCC) and provide a permissible environment for chronic infection and inflammation (11-17). While knowledge of disease progression in CF and other obstructive lung diseases has grown substantially over the past few decades, the critical biophysical properties of airway mucus that allow maintenance of lung function have yet to be determined. Additionally, the role of mucus abnormalities on bacteria- and/or virus-mediated pulmonary exacerbations in obstructive lung diseases remains unclear.

Sputum samples collected from CF patients have been studied extensively to gain a basic understanding of the role of mucus in the progression of CF lung disease. CF airway mucus has markedly higher viscosity and elasticity than mucus collected from healthy individuals $(18,19)$. Recent studies in $3 \mathrm{D}$ human airway cultures have established that hyperconcentrated mucus impairs MCC, leading to mucus accumulation on airway epithelium (20). Based on this finding, percent solid content of mucus has been identified as a candidate marker for disease progression in obstructive lung diseases (21). In a recent study, 
the solid content in airway mucus was negatively correlated with lung function in chronic bronchitis (CB), an obstructive lung disease sharing mucus pathology similar to CF (22). It was also recently found that oxidative stress resulting from chronic lung inflammation can increase mucin disulfide cross-linking that further stiffens the mucus gel and enhance its viscoelasticity (23). On the microscale level, the mucus gel consists of polymeric mucins and other biomacromolecules that organize through disulfide cross-linking and/or physical entanglements to form a mesh-like structure, which we refer to as microstructure. The bulk properties of airway mucus do not capture microscale mucus mesh structure relevant to the growth and establishment of infection or the response of immune cells to infection $(24,25)$. We hypothesize that the changes in sputum microstructure, on the length scales of viruses, bacteria, and neutrophils, will contribute to more frequent pulmonary exacerbations and accelerated decline in lung function in CF.

We have shown previously that the microrheological and microstructural properties of human mucus secretions can be assessed by monitoring the diffusion rates of muco-inert nanoparticle (MIP) probes, also referred to as mucus-penetrating particles (MPP) in our prior studies (26-30). MIP possessing diameters smaller than mucus mesh spacings are capable of efficiently penetrating sputum, and thus were referred to as MPP. Due to the dense surface coatings with polyethylene glycol (PEG) that render the particle surface muco-inert, MIP transport in mucus is primarily hindered by physical obstruction imposed by the mucus mesh structure rather than adhesive interactions $(26,31)$. Thus, microstructural properties, specifically mucus mesh pore size, can be quantitatively determined by measuring transport of MIP in sputum. We recently found that the variance in transport rates of 100-nm MIP in sputum samples from different CF patients was approximately 50 -fold greater than the variance among multiple sputum sample aliquots from the same individual patients (31). This suggests that microstructural properties of CF sputum are patientspecific, irrespective of intrasample heterogeneity. In this study, we evaluated the relationship between biochemical content of CF sputum and the microstructural properties of sputum from individual CF patients. We further assessed the clinical implications of varied sputum microstructure by directly correlating with patients' lung function, as measured via spirometry, as well as by analyzing the effect of ex vivo treatment of CF sputum with recombinant human DNase I (rhDNase I; Pulmozyme). The results of this study establish a method to assess the micro- and nanoscale properties of sputum and yield insights into the progression of CF lung disease.

\section{Results}

Effect of CF sputum microstructure on nanoparticle transport. To visualize patient-to-patient differences in sputum microstructure, we revealed the structure of the mesh by staining the fluid-filled pores in sputum with fluorescent dextran. As a representative case, two patient samples were compared using this method. The differences in microstructure of the 2 samples were clear, with much larger pores (dark regions in Figure 1, A-D) apparent in sputum from patient 1 compared with that from patient 2 (Figure 1, A and B). There was also an approximately 10 -fold higher measured void volume fraction (i.e., porosity) in the sputum sample from patient 1 as compared with that from patient 2 (Figure 1, A and B).

Our previous studies have established that 100-nm MIP avoid adhesive interactions with sputum components, and, thus, their movement is primarily hindered by steric interactions with the sputum mesh network $(26,31)$. Thus, measured differences in 100-nm MIP transport rates among individual patients' sputum samples should correspond to differences in their sputum porosity. To confirm this hypothesis, multiple particle tracking (MPT) (32) was used to measure the transport of 100-nm MIP within the center of the same regions of each sample shown in Figure 1, A-D. 100-nm MIP travel over much longer distances in sputum from patient 1 versus patient 2, as shown in representative trajectories (Figure 1, E and F). Quantitative MPT analysis of $>500$ particles also indicated that the logarithm (based 10) of mean squared displacement (MSD) per $\mu \mathrm{m}^{2}$ at time scale $\tau=1 \mathrm{~s}\left(\log _{10}\left[\mathrm{MSD}_{1 \mathrm{~s}}\right]\right)$, a measure of nanoparticle transport rate, is reduced in patient 2 in comparison with that in patient 1 (Figure 1, G and $\mathrm{H}$ ).

Patient-to-patient differences in sputum microstructural properties. We next compared the $\log _{10}\left[\mathrm{MSD}_{1 \mathrm{~s}}\right]$ of 100 $\mathrm{nm}$ MIP of CF sputum samples among 10 patients as a potential patient-specific marker of sputum microstructural properties. We found that the $\log _{10}\left[\mathrm{MSD}_{1 \mathrm{~s}}\right]$ varied among $\mathrm{CF}$ patients, with median values ranging from -2 to 0.3 (Figure 2A). For comparison, the $\log _{10}\left[\mathrm{MSD}_{1 \mathrm{~s}}\right]$ of $100-\mathrm{nm}$ MIP in mucus collected from 5 non-CF individuals was also measured (Figure 2A). Unlike CF patients, non-CF individuals cannot spontaneously produce sputum samples, and, thus, the mucus samples were collected from endotracheal (ET) tubes used during general anesthesia in an operating room. For a physical interpretation of $\log _{10}\left[\mathrm{MSD}_{1 \mathrm{~s}}\right]$, 
Patient 1

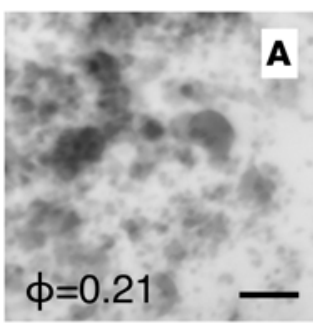

Patient 2

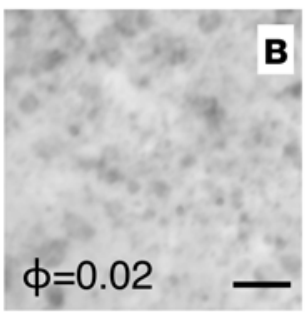

B

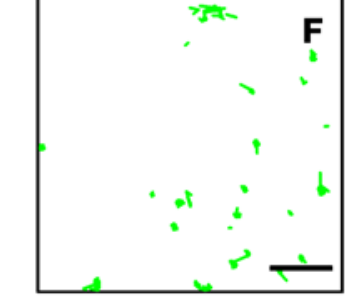

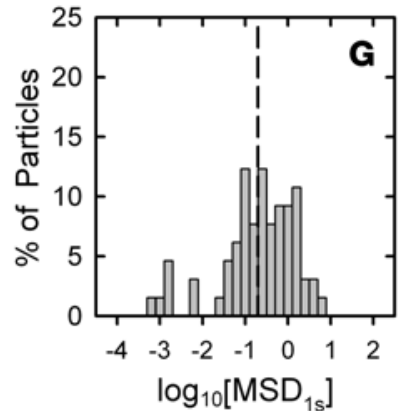

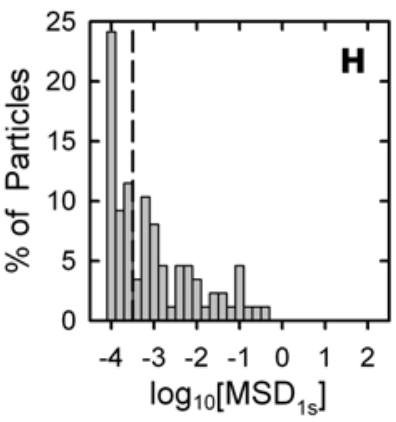

Figure 1. Change in 100-nm muco-inert nanoparticle transport due to changes in cystic fibrosis sputum microstructure. (A-D) 3D confocal imaging of sputum microstructure in individual cystic fibrosis (CF) patient samples using the dextran-negative staining method (black, void space; white, solid matrix). (A and B) 3D $x y$ projections and ( $C$ and $\mathbf{D}) 2 \mathrm{D} x z$ profiles generated from 25 images with $0.33-\mu \mathrm{m}$ spacing with measured void volume fraction (porosity $[\phi]$ ) shown (scale bar: $4 \mu \mathrm{m}$ ). (E-H) Multiple particle tracking of 100-nm muco-inert nanoparticles (MIP) within CF sputum samples from patient 1 ( $\mathbf{E}$ and $\mathbf{G}$ ) and patient $2(\mathbf{F}$ and $\mathbf{H})$ in the regions shown in A-D. (E and F) 2D trajectories of 100-nm MIP in each CF sputum sample at plane marked by dashed green line indicated in $x z$ profiles from confocal images shown in $\mathbf{C}$ and $\mathbf{D}$ (scale bar: $4 \mu \mathrm{m}$ ). ( $\mathbf{G}$ and $\mathbf{H})$ Distribution of individual particles' log base 10 of MSD per $\mu \mathrm{m}^{2}$ at time $\tau=1 \mathrm{~s}\left(\log _{10}\left[\mathrm{MSD}_{15}\right]\right)$ in for $100-\mathrm{nm}$ MIP in each sputum sample. Dashed lines indicate median values.

we calculated the complex microviscosity $\left(\eta^{*}\right)$, a quantity that reflects the viscosity experienced by the nanoparticles on the microscale (26). We report microviscosity at $\omega=1 \mathrm{rad} / \mathrm{s}$ as low frequency measurements, corresponding to the time scale of mucus transport via MCC, which are most often used in mucus rheology studies $(33,34)$. The median value of complex microviscosity varied over sev-

eral orders of magnitude, ranging from $1 \times 10^{-2}$ to $2 \times 10^{0} \mathrm{~Pa} \cdot \mathrm{s}$ (Figure $2 \mathrm{~B}$ ). In addition, we measured viscosity of interstitial fluid (ISF) from CF sputum $\left(\eta_{\text {ISF }}\right)$, and it was approximately equal for $n=4$ patients $\left(\eta_{\text {ISF }}=8.28\right.$ $\times 10^{-3} \pm 1 \times 10^{-4} \mathrm{~Pa} \cdot \mathrm{s}$; dashed line in Figure $\left.2 \mathrm{~B}\right)$. The estimated median pore size of each sputum sample ranged from 110 to $930 \mathrm{~nm}$ (Figure 2C, gray bars), consistent with prior measurements $(26,35)$. Complex microviscosity and pore size were also determined for non-CF mucus samples (Figure 2, B and C). We additionally investigated the impact of MIP size on their transport in CF sputum to determine the range of mesh spacings that can be detected using the MPT method. Based on the range of CF sputum pore sizes previously estimated (60-300 nm) $(26,35)$, we quantified the diffusion rates of 100-, 200-, and 500-nm MIP in CF sputum and found that transport of 100-nm MIP most sensitively differentiated sputum samples based on patient clinical status (Supplemental Figure 1A; supplemental material available online with this article; doi:10.1172/ jci.insight.88198DS1). In addition, we assessed 100-nm MIP transport in 3 repeated collections of sputum samples from an individual patient with approximately 10-minute intervals between expectorations and found no statistically significant differences in $\log _{10}\left[\mathrm{MSD}_{1 \mathrm{~s}}\right]$ between samples (Supplemental Figure 1B).

Biochemical composition of sputum and its impact on microstructure. In order to determine the effects of key biochemical components of CF sputum on microstructure, we quantitatively measured the percent solids, mucin, DNA, and cystine (disulfide bridge) contents of CF sputum. Each of these measures was negatively correlated with statistical significance to the median MIP transport rate (median $\log _{10}\left[\mathrm{MSD}_{1 \mathrm{~s}}\right]$ ) (Figure 3 ) where DNA content correlated most strongly $\left(R^{2}=0.41, P=0.003\right)$, followed by mucin content $\left(R^{2}=0.31, P=0.02\right)$, percent solids content $\left(R^{2}=0.23, P=0.03\right)$, and cystine content $\left(R^{2}=0.21, P\right.$ $=0.04)$. Further, we performed principal component analysis to determine the key contributing factors to variation in $\log _{10}\left[\mathrm{MSD}_{1 \mathrm{~s}}\right]$, mucin, DNA, total solids, and cystine content between sputum samples. We found that increases in mucin, DNA, and/or total solids content were well correlated with decreases in median $\log _{10}\left[\mathrm{MSD}_{1 \mathrm{~s}}\right]$ (i.e., reduced sputum pore sizes) (Supplemental Tables 1 and 2).

Microstructural properties of artificial sputum hydrogels. To mechanistically understand the effects of biochemical alterations on sputum microstructure, we assessed how the cross-linking density and DNA concentration, which are elevated in $\mathrm{CF}(8,23)$, affected microstructure in a model mucin-based hydrogel system. Mucin-based hydrogels were produced by cross-linking mucin fibers using PEG-diacrylate (PEGDA), and the resulting bulk viscoelastic properties of the model mucus gel were confirmed qualitatively by an inversion test (Figure 4A). As evidenced by 100-nm MIP transport rates in mucin-based hydrogels at a fixed 


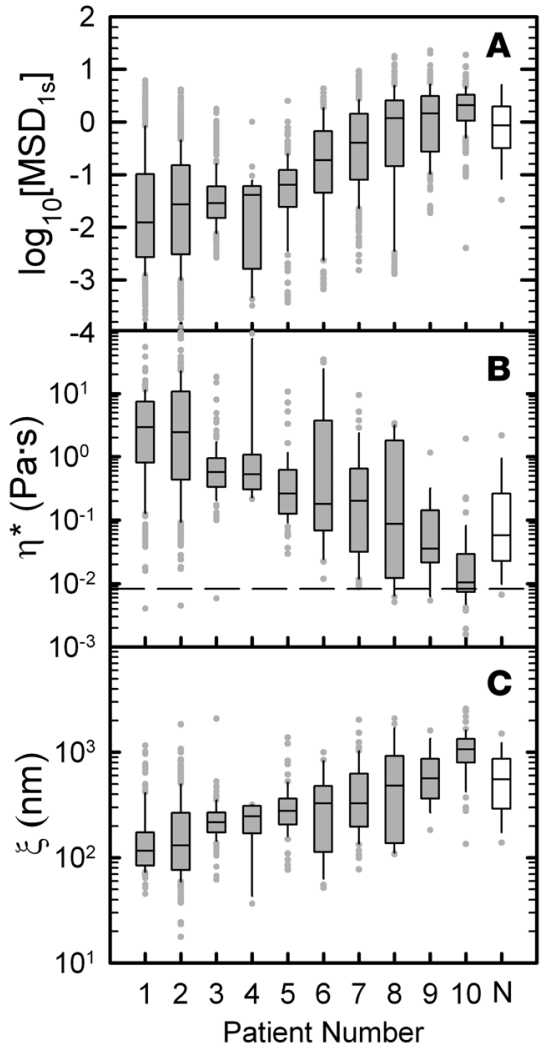

Figure 2. 100-nm muco-inert nanoparticle transport is sensitive to cystic fibrosis patient-specific sputum microstructural properties. Box-and-whisker plots of log base 10 of MSD per $\mu \mathrm{m}^{2}$ at time $\tau=1 \mathrm{~s}\left(\log _{10}\left[\mathrm{MSD}_{1 \mathrm{~s}}\right]\right)$ of $100 \mathrm{~nm}$ muco-inert nanoparticles (MIP) in sputum samples from 10 cystic fibrosis (CF) patients (gray bars). The $\log _{10}\left[\mathrm{MSD}_{15}\right]$ of $100-\mathrm{nm}$ MIP in mucus samples collected from 5 non-CF (N) individuals via an ET tube method is also included for comparison (white bars). A microrheological analysis of MSD was used to determine complex microviscosity $\left(\eta^{*}\right)$ and mesh size $(\xi)$ for each subject. The maximum whisker length is 1.5 times the interquartile range and outliers are shown as dots. CF patients are numbered in ascending order according to median $\mathrm{MSD}_{15}$ of $100-$ nm MIP. (A) Measured $\log _{10}\left[\mathrm{MSD}_{15}\right]$ of 100-nm MIP in CF sputum and non-CF mucus samples. (B) Calculated $\eta^{*}$ at $\omega=1 \mathrm{rad} / \mathrm{s}$ from microrheological analysis of measured MSD. $\eta^{*}$ for CF sputum interstitial fluid is indicated by the dashed line and was characterized by centrifuging 4 sputum samples at $21,000 \mathrm{~g}$ for 1 hour and measuring the supernatant's viscosity by MPT. (C) Estimated mesh pore sizes of CF sputum and non-CF mucus samples calculated as $\xi \approx \sqrt[3]{ } k_{B} T I G^{\prime}$ based on measured elastic modulus ( $\left.G^{\prime}\right)$ at $\omega=1 \mathrm{rad} / \mathrm{s}$ from microrheological analysis of measured MSD. Statistical analyses comparing CF sputum and non-CF mucus were not conducted because samples were collected using different methods (i.e., spontaneous expectoration vs. ET tube method).

solids content of $5 \%$, increasing the PEGDA concentration led to an increase in crosslinking density of mucin-based hydrogel, thereby reducing mesh pore size (Figure 4B). In addition, an increase in the DNA concentration in mucin/DNA composite hydrogels, at a fixed percent solids content of $5 \%$, resulted in marked reductions in mesh pore sizes (Figure 4C). We also found that treating hydrogels at a solids/mucin/DNA composite content of $5 \%$ with rhDNase at a final concentration of $7 \mu \mathrm{g} / \mathrm{ml}$ effectively reversed the reduction in pore sizes to the baseline value of hydrogels containing mucin only, at the same overall percent solids content (Figure 4D).

Clinical significance of CF sputum microstructure. We next assessed whether patient-specific sputum microstructural properties correlate with clinical status. The demographics of patients included in this study are shown in Table 1 . The median $\log _{10}\left[\mathrm{MSD}_{15}\right]$ measurements of 100-nm MIP were compared with measured forced expiratory volume after $1 \mathrm{sec}-$ ond $\left(\mathrm{FEV}_{1}\right)$, the primary measurement for pulmonary function and lung disease severity in $\mathrm{CF}(36)$. Based on a cross-sectional analysis of 25 patients, median $\log _{10}\left[\mathrm{MSD}_{1 \mathrm{~s}}\right]$ positively correlated with measured $\mathrm{FEV} \mathrm{V}_{1}$ $\left(R^{2}=0.45\right)$ and was statistically significant $(P<0.001$; Figure 5A). In contrast, percent solids content was not significantly correlated with $\mathrm{FEV}_{1}\left(R^{2}=0.01\right.$ and $P=0.60$; Figure $\left.5 \mathrm{~B}\right)$. We also conducted a longitudinal analysis of sputum samples collected from $5 \mathrm{CF}$ patients at the first and subsequent visits to the clinic, with an interval of approximately 3 months. We found that change in median $\log _{10}\left[\mathrm{MSD}_{1 \mathrm{~s}}\right]\left(\log _{10}\left[\mathrm{MSD}_{1 \mathrm{~s}}\right]\right)$ was proportional to that in $\mathrm{FEV}_{1}\left(\triangle \mathrm{FEV}_{1}\right.$; Figure $\left.5 \mathrm{C}\right)$. We compared $\log _{10}\left[\mathrm{MSD}_{15}\right]$ among patients based on age, gender, delF508 mutation type (i.e., heterozygous vs. homozygous), CF-related diabetes status, and approximate time since patients' most recent Pulmozyme inhalation and found no significant difference in median $\log _{10}\left[\mathrm{MSD}_{1 \mathrm{~s}}\right]$ based on any of these factors (Supplemental Figure 2). We also compared the median $\log _{10}\left[\mathrm{MSD}_{15}\right]$ among patients based on current pulmonary infections, and we found that 3 patients infected with both Pseudomonas aeruginosa and methicillinresistant Staphylococcus aureus (MRSA) showed lreduced sputum pore sizes compared with 6 patients with MRSA only (Supplemental Figure 2E). However, there were no significant differences among 14 patients with $P$. aeruginosa-only infection versus those with $M R S A$ or those coinfected with P. aeruginosa and MRSA (Supplemental Figure 2E).

Figure 3. Impact of cystic fibrosis (CF) sputum biochemical composition on microstructure. The correlations of $\log$ base 10 of the median MSD per $\mu \mathrm{m}^{2}$ at time $\tau=1 \mathrm{~s}$ (median $\log _{10}\left[\mathrm{MSD}_{15}\right]$ ) of $100-\mathrm{nm}$ MIP measured in sputum samples from 18 CF patients versus (A) percent solids, (B) mucin, (C) DNA, and (D) cystine (disulfide bond) content of each sputum sample. $R^{2}$ values were determined based on a linear regression analysis, and the correlations were considered statistically significant when $P<0.05$.

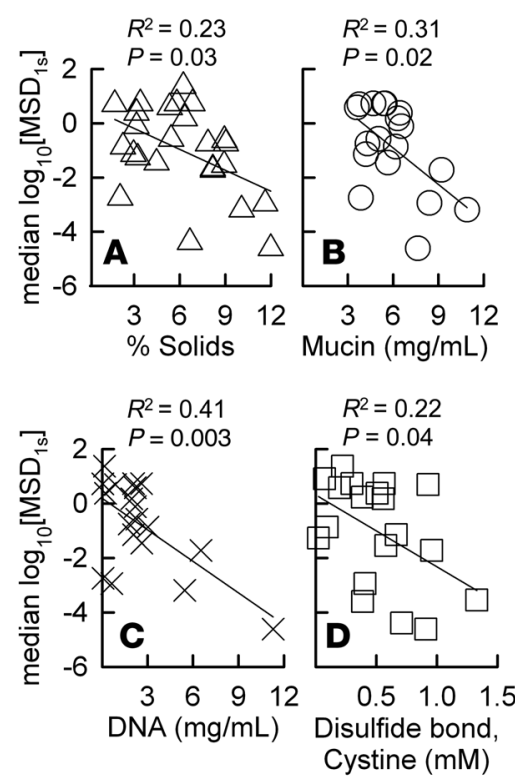



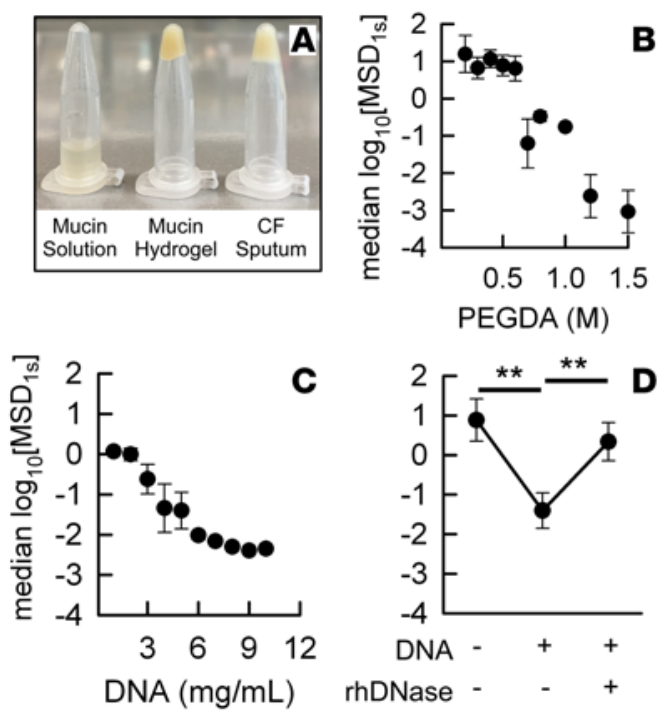

Figure 4. Mechanistic study of microstructure in cross-linked mucin-based hydrogels. (A) Inversion test showing bulk viscoelastic properties of a mucin solution or a crosslinked mucin-based hydrogel, both containing $5 \%$ solids, and a CF sputum sample (right). The correlation of median $\log _{10}\left[\mathrm{MSD}_{15}\right]$ versus (B) PECDA cross-linking density in mucinbased hydrogels or (C) DNA content in mucin/DNA composite hydrogels, both at a fixed percentage solids content of $5 \%$. (D) $\log _{10}\left[\mathrm{MSD}_{15}\right]$ measured in $5 \%$ mucin only hydrogel, mucin/DNA composite ( $4.5 \%$ mucin + 0.5\% DNA) hydrogel, and the composite hydrogel after a 30 -minute incubation with $7 \mu \mathrm{g} / \mathrm{ml}$ rhDNase at $37^{\circ} \mathbf{C}$. Data in $\mathbf{B}-\mathbf{D}$ represents the average of 3 independent hydrogel preparations and the microstructural analysis of each. Student's $t$ test was used to compare different conditions in $\mathbf{D}\left({ }^{* *} P<0.01\right)$.

Effect of rhDNase on CF sputum microstructure. As rhDNase is one of the most widely used mucolytics in CF (37), we sought to determine the effect of rhDNase treatment on the microstructure of CF sputum. After collection, sputum samples were treated with rhDNase at a final concentration of $7 \mu \mathrm{g} / \mathrm{ml}$, the estimated concentration in sputum after nebulization $(38,39)$. In a representative patient sample, the porosity of CF sputum increased by 3-fold after treatment with rhDNase compared with the PBS-treated control (Figure 6, A and B). For this representative case, the patient had not taken rhDNase within the past 24 hours prior to the spu-

tum collection. However, for a cohort of 9 patients, we saw marked differences in ex vivo response to rhDNase treatment based on measured $\log _{10}\left[\mathrm{MSD}_{1 \mathrm{~s}}\right]$ (Figure 6C). By further controlling for each patient sample based on their most recent inhaled treatment with rhDNase, we found that patients who had inhaled rhDNase $\geq 24$ or $12-16$ hours prior to the sputum collection robustly responded to the ex vivo rhDNase treatment, with an increase in $\log _{10}\left[\mathrm{MSD}_{1 \mathrm{~s}}\right]$ that indicates an enlargement of mucus mesh pore size. In contrast, patients that had taken rhDNase 2-6 hours prior to the sputum collection had a very limited response to ex vivo rhDNase treatment, with no statistically significant change in $\log _{10}\left[\mathrm{MSD}_{1 \mathrm{~s}}\right]$. The transport rate of MIP in ISF, which is proportional to $\eta_{\text {ISF }}$, was approximately equal before and after the ex vivo treatment with rhDNase based on the measured $\log _{10}\left[\mathrm{MSD}_{1 \mathrm{~s}}\right]$ (Supplemental Figure 3).

\section{Discussion}

Little is known about the microscale architecture of CF sputum and its potential implications in disease progression. Thus, we have developed a sensitive assay capable of detecting the microstructure of unperturbed sputum samples freshly collected from individual CF patients. We found that a reduction in transport rates of 100-nm MIP in CF sputum, indicating a tightening of the sputum mesh pore size, correlated with lung disease severity signifed by decreased $\mathrm{FEV}_{1}$. We also demonstrated that the pore size within the $\mathrm{CF}$ sputum mesh increased after treatment with the most widely used mucolytic agent, rhDNase. Based on our results, sputum microstructure may serve as a useful biophysical marker for CF lung disease and as a novel outcome measure to evaluate mucusaltering therapeutic agents. Importantly, we used a fully automated, high-throughput method for analysis of sputum microstructure, allowing for multiple samples collected from patients visiting the clinic to be assessed within a short time period.
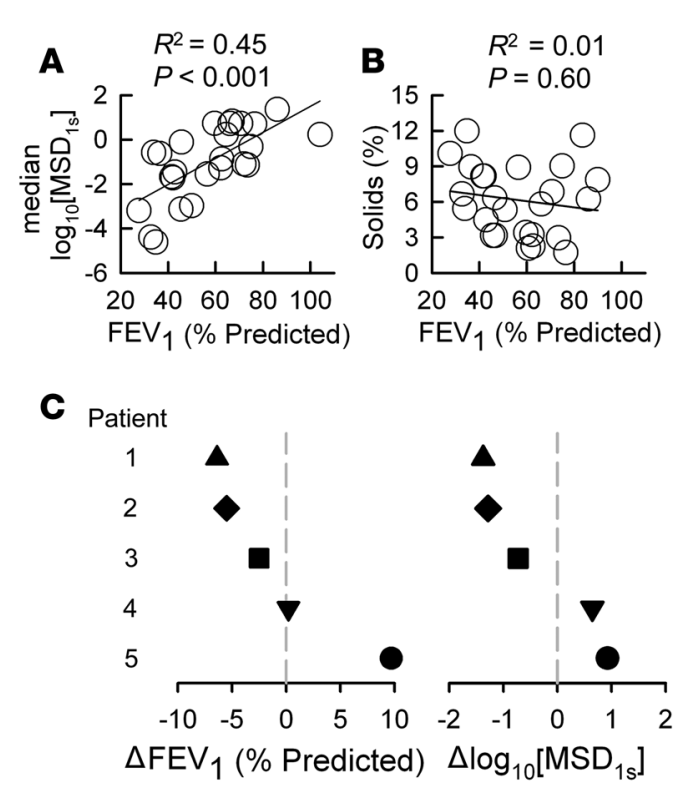

Figure 5. Clinical significance of cystic fibrosis sputum microstructure. The correlation of (A) median $\log _{10}\left[\mathrm{MSD}_{15}\right]$ or (B) percent solids content, measured in sputum samples from 25 cystic fibrosis (CF) patients, versus measured forced expiratory volume after 1 second $\left(\mathrm{FEV}_{1}\right)$. FEV was measured from patients prior to sputum collection. The $R^{2}$ values were determined based on the linear regression analysis, and the correlations were considered statistically significant when $P<0.05$. (C) Sputum samples were collected from the same patients twice, once initially and again approximately 3 months later. The change in measured $F E V_{1}$ and in $\log _{10}\left[\mathrm{MSD}_{15}\right]\left(\Delta \log _{10}\left[\mathrm{MSD}_{15}\right]\right)$ between the first and second collection is shown. Dashed line indicates $\Delta \log _{10}\left[\mathrm{MSD}_{15}\right]=0$ or $\Delta \mathrm{FEV}_{1}=0$ (i.e., no change in microstructure or $\mathrm{FEV}_{1}$, respectively). 


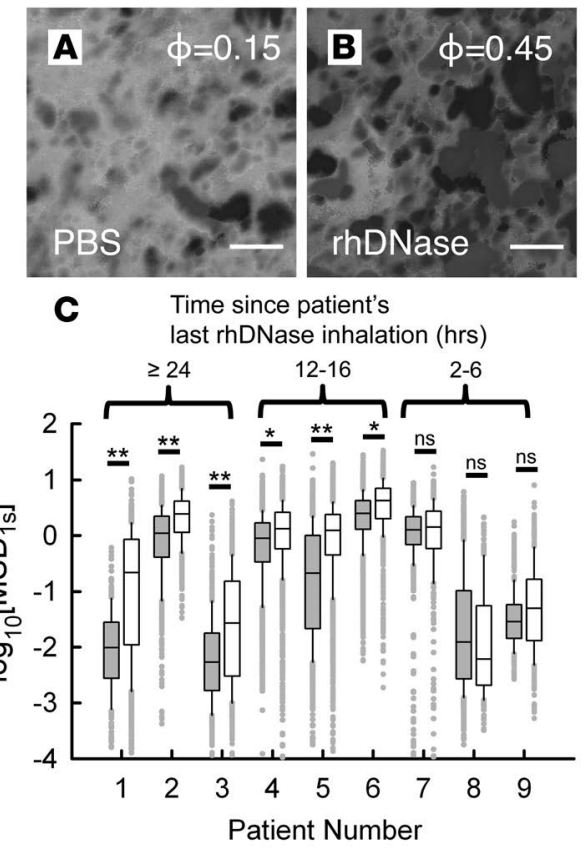

Figure 6. Effect of rhDNase treatment on cystic fibrosis sputum microstructure. (A and B) 3D $x y$ projections from confocal imaging of a cystic fibrosis (CF) sputum sample with and without ex vivo rhDNase treatment at final concentration of $7 \mu \mathrm{g} / \mathrm{ml}$ (scale bar: $2 \mu \mathrm{m})$. Measured void volume fraction or porosity $(\phi)$ is also shown in each image. The patient's last Pulmozyme administration was $>24$ hours before sputum collection. (C) Box-and-whisker plots of $\log _{10}\left[M_{15} D_{15}\right.$ of $100-n m$ muco-inert nanoparticles (MIP) in sputum samples from 9 CF patients without (gray bars) and with (white bars) ex vivo rhDNase treatment at a final concentration of $7 \mu \mathrm{g} / \mathrm{ml}$. Maximum whisker length is 1.5 times the interquartile range, and outliers are shown as dots. The $\log _{10}\left[\mathrm{MSD}_{15}\right]$ values are grouped based on the approximate time interval between the patients' most recent Pulmozyme inhalation and the sputum collection. Mann-Whitney test was used to determine statistically significant differences between rhDNase-treated and untreated controls for each patient (not significant, $P>0.05 ;{ }^{*} P<0.05 ;{ }^{* *} P<0.01$ ).

We have shown in prior studies that dense surface coatings with PEG on nanoparticles minimize their adhesion to sputum constituents, and the rates at which they move within sputum primarily depend on the degree of physical obstruction imposed by the nanoporous sputum mesh $(26,31)$. Without a muco-inert coating, the diffusion of nanoparticles is restricted by the sputum microstructure as well as adhesive interactions of nanoparticles with components in sputum via electrostatic and hydrophobic interactions $(40,41)$. The tighter sputum microstructure revealed by slower 100-nm MIP diffusion was validated by lower porosity $(\phi)$ determined via a newly developed negative fluorescence staining method. However, this staining technique, while providing visual evidence, is limited in detecting small pores

$(<120 \mathrm{~nm})$ due to the microscope's resolution and thus does not provide precise, quantitative information on sputum microstructure as can be achieved with the MPT-based method. We additionally found 100-nm MIP transport in CF sputum to be the most sensitive to patient clinical status in comparison to 200-nm and 500-nm MIP. Thus, 100-nm MIP transport was used throughout this study as the primary measurement of CF sputum microstructure.

Microstructural properties of airway mucus secretions, in particular the tightening of the mucus mesh, could play a significant role in sustained infections and recurrent exacerbations characteristic of CF lung disease. Reduction in pore size in CF airway mucus can act to slow the migration of neutrophils and inhibit their activity against infection. A prior study demonstrated this effect in mucin hydrogels, in which neutrophil migration and bacterial capture is inhibited in highly concentrated mucin gels (24). We also expect reduced pore sizes to enhance bacterial colonization by inhibiting bacterial motility in mucus from sites of replication, as daughter cells will be trapped within the gel. In support of this hypothesis, a prior study showed that hindered motility of $P$. aeruginosa in CF-like mucin hydrogels promotes growth of biofilms (25). The combined effects of neutrophil inhibition and biofilm production in the CF lung are likely to contribute to the cycle of chronic inflammation, infection, and deterioration of lung function. In a recent study on COPD sputum, we found a similar trend of sputum mesh tightening in COPD patients with severe airway obstruction (our unpublished observations), suggesting a similar role of sputum microstructure in other obstructive lung diseases.

The accumulation of hyperconcentrated sputum in the airways has long been established as a hallmark of CF lung disease $(7,42)$. Recent studies have clearly demonstrated the effect of airway mucus concentration on MCC, with elevated concentrations causing osmotic pressure-driven periciliary layer (PCL) collapse (20). Based on this finding, the percent solids content has been assessed as a biophysical marker for lung disease severity, and it was found to negatively correlate with lung function of

\section{Table 1. Patient demographics}

\begin{tabular}{lc}
\hline No. patients & $\mathbf{3 1}$ \\
$\begin{array}{l}\text { Age (yr; mean } \pm \text { SD) } \\
\% \text { Predicted FEV }\end{array}$ (mean & $34 \pm 10$ \\
\pm SD) & \\
Cender (\%) & \\
Male & 52 \\
Female & 48 \\
\hline CFTR genotype (\%) & \\
delF508 homozygous & 55 \\
delF508 heterozygous & 45 \\
Other & 0 \\
CFRD status (\%) & \\
Yes & 19 \\
No & 67 \\
Unknown & 14 \\
Infection type (\%) & \\
P. aeruginosa & 45 \\
MRSA & 19 \\
Other & 36
\end{tabular}

$\mathrm{FEV}_{1}$, forced expiratory volume after 1 second; CFTR, cystic fibrosis transmembrane conductance regulator; CFRD, cystic fibrosis-related diabetes; MRSA, methicillin-resistant S. aureus. 
$\mathrm{CB}$ patients (22). However, we found that the percent solids content of CF sputum samples did not significantly correlate with $\mathrm{FEV}_{1}$. Thus, the observed trend in reduced lung function may not be fully explained by impaired MCC, resulting from the increased percent solids content. It should be also noted that the mechanisms by which lung function declines, particularly involving mucus properties, may vary depending on the type of obstructive lung diseases (i.e., CF versus CB). We should, however, note that cross-sectional studies monitoring these parameters at a single point may not fully reflect how they progressively change in CF lung disease, as is ascertained with longitudinal studies.

While measurements of sputum biochemical composition may be more straightforward, the spatial arrangement of biomolecules in sputum (i.e., microstructure) could provide new critical insights into the CF lung microenvironment. Biochemical analysis revealed that increases in mucin, DNA, percent solids, and disulfide bond content all reduced the size of pores within the sputum mesh. Further, we have mechanistically demonstrated that mucin cross-linking density and DNA concentration can affect the mesh pore size of mucin-based hydrogels with a fixed percent solids content, suggesting that the microstructural properties of mucus are not controlled by solids content alone. The resulting changes in sputum microstructure are most likely dictated by a multitude of factors, including mucin hyperconcentration $(17,42)$, physical entanglements between mucin and DNA $(13,23,43)$, and/or enhanced mucin disulfide cross-linking (23). Our measurements reflect their collective contributions to sputum microstructure.

We also found that ex vivo rhDNase treatment significantly increased pore sizes of CF sputum. However, this phenomenon was dependent upon the time interval between the patients' most recent rhDNase inhalation and sputum expectoration. Specifically, the minimal effects of ex vivo rhDNase treatment observed with short time intervals suggest that the prior rhDNase inhalation left DNA present in the sample degraded into fragments, thus limiting the effects of further rhDNase treatments. Our findings may provide indirect evidence of in vivo effects of inhaled Pulmozyme on the biophysical properties of CF sputum. Previous studies by our group and others found that ex vivo rhDNase treatment had a minimal impact on nanoparticle transport through CF sputum, implying negligible change in sputum microstructure after treatment $(39,43,44)$. The discrepancy most likely arises from the use of nanoparticles without muco-inert coatings in prior studies, which resulted in a significant contribution of adhesive interactions with sputum components on their transport rate. Additionally, some of these earlier studies did not provide information related to patients' prior rhDNase treatment. Overall, our results suggest that the clinical benefit of rhDNase and other mucolytic agents may involve their modulatory effect on sputum microstructure.

A limitation of our assay is the necessity of fresh, undiluted CF sputum in order for samples to retain their physiological microstructure (26). We thus used spontaneously expectorated sputum samples stored less than 24 hours at $4^{\circ} \mathrm{C}$ for all of our studies. Induced $\mathrm{CF}$ and/or non-CF sputum samples were not used, as sputum may not retain its physiological microstructure due to dilution by inhaled saline. As a result, we excluded CF patients who were unable to spontaneously produce sputum. In addition, we did not account for individual patients' clinical status during their visits, specifically concerning whether disease symptoms were stable or had worsened compared with baseline. Our future studies will assess the microstructure of sputum samples collected before and after acute decline of lung function in order to determine if microstructure may serve as a novel marker or predictor of pulmonary exacerbation in CF.

Based on the findings from this study, it is unclear whether the observed changes in sputum microstructure directly contribute to disease progression or if microstructural alterations occur as a result of CF lung disease. We have suggested potential mechanisms to explain how microstructure could affect infection and inflammation in the CF lung. In future studies, we will assess the migration of neutrophils and bacteria in model mucus hydrogels with controlled composition to mechanistically study the effect of microstructure on $\mathrm{CF}$ and other obstructive lung diseases. It is also unknown how the microstructure of mucus may be involved in its clearance from the airway. Studies on the effect of sputum/mucus microstructure on its interactions with the PCL, in terms of mucus adhesion to the PCL and interpenetration between the PCL and mucus gel layer, have been limited to date. However, interactions at the mucus-PCL interface on these length scales could also contribute to $\operatorname{MCC}(12,45)$ and warrant further investigation.

In summary, our findings show that the biophysical alteration of sputum on the micro- to nanoscale could serve as an indicator of CF lung disease progression. Based on the transport of MIP in CF sputum, the size of pores in the sputum mesh can be assessed to determine patient-specific "microstructural" phenotypes. Alteration of sputum microstructure, more specifically a tightening of pores within the sputum mesh network, is characteristic of CF patients with reduced lung function. In a small cohort of CF patients, 
improvement or decline in lung function trended proportionally with increases or decreases in CF sputum mesh pore sizes, respectively. Our findings motivate future longitudinal studies with frequent assessments (i.e., daily or weekly) of a large CF patient cohort to determine if mucus microstructural properties may serve as a novel biophysical marker of CF lung disease progression.

\section{Methods}

Preparing PS-PEG (muco-inert) nanoparticles. PEG-coated polystyrene particles (PS-PEG) were prepared as detailed previously (27). Using this protocol, the high surface density of PEG renders nanoparticles "mucoinert," indicating that they are effectively shielded from adhesive interactions with the biomolecular components of CF sputum $(27,31,35)$. Briefly, red fluorescent carboxylate-modified PS spheres (PS-COOH), $100 \mathrm{~nm}$ in diameter (Life Technologies), were coated with 5-kDa methoxy-PEG-amine (Creative PEGWorks) via covalent attachment to $\mathrm{COOH}$ groups on the PS-COOH particles using 1-ethyl-3-(3-dimethylaminopropyl) carbodiimide hydrochloride (Sigma-Aldrich) and N-hydroxysulfosuccinimide sodium salt (Sigma-Aldrich) in borate buffer ( $\mathrm{pH}$ 8.3). The reactant mixture was incubated for at least 4 hours at room temperature. The PS-PEG particles were then centrifuged and washed 3 times in ultrapure water.

Preparing mucin-based hydrogels. Hydrogels primarily composed of porcine gastric mucin (PGM; SigmaAldrich) were prepared by addition of $600-\mathrm{kDa}$ PEGDA (Sigma-Aldrich) to PGM solutions. PEGDA has been used extensively in prior studies to form hydrogels composed of thiolated biopolymers (46-48). PGM solutions with a concentration of $4 \%(\mathrm{w} / \mathrm{v})$ were prepared in a buffer solution containing $154 \mathrm{mM} \mathrm{NaCl}, 3$ $\mathrm{mM} \mathrm{CaCl}_{2}$, and $15 \mathrm{mM} \mathrm{NaH}_{2} \mathrm{PO}_{4}$ at $\mathrm{pH} 7.4$ and were rapidly mixed for at least 2 hours using a magnetic stir plate. PEGDA at varying final concentrations were added dropwise to PGM solutions to achieve a final concentration of $2 \%(\mathrm{w} / \mathrm{v})$. The mixed solution of PEGDA and PGM was incubated for 1 hour at room temperature to allow cross-linking to occur. The PEGDA cross-linked mucin was subsequently collected by centrifugation at 21,000 $\mathrm{g}$ for 1 hour. The supernatant was removed, and the pellet was then flash frozen in liquid $\mathrm{N}_{2}$ and lyophilized overnight. Prior to mixing with lyophilized, cross-linked PGM, buffer solutions containing varying amounts of DNA from salmon testes (Sigma-Aldrich) were prepared by rapidly mixing for at least 2 hours using a magnetic stir plate. Lyophilized, cross-linked mucins were reconstituted to the final overall solids content of $5 \%$ in the original buffer solution only or in the buffer solution containing DNA. PGM-only and/or PGM/DNA composite hydrogels were then allowed to mix on a rotating stirrer for 24 hours before being used in experiments.

$C F$ sputum sample collection. Spontaneously expectorated sputum samples were collected from patients at the adult CF clinic at Johns Hopkins $(n=31)$. Samples were stored at $4^{\circ} \mathrm{C}$ immediately after collection for up to 24 hours for confocal imaging and particle tracking experiments. We have confirmed that the microstructural properties of freshly collected sputum are maintained when samples are stored for 24 hours at $4^{\circ} \mathrm{C}$ (Supplemental Figure 4). After 24 hours, sputum samples were stored at $-80^{\circ} \mathrm{C}$ and thawed on ice for any additional biochemical analyses. Age, gender, CF mutation, day/time of last use of rhDNase, FEV from that day, and results of sputum bacterial culture from that day were recorded. Patients involved in this study received no mucolytics other than Pulmozyme and/or hypertonic saline as part of their standard treatment regimen. In an effort to reduce the effects of salivary contamination, sputum samples with visible quantities of saliva were not included in the analysis.

Non-CF mucus sample collection. Samples were collected by the ET tube method, as previously described (27). Briefly, ET tubes were collected from patients after intubation as a part of general anesthesia for elective, noncardiothoracic surgery at the Johns Hopkins Hospital. Only patients with no cardiopulmonary or respiratory comorbidities and no smoking history were included in this study. In order to collect mucus from ET tubes, the last approximately $10 \mathrm{~cm}$ of the tubes were cut, including the balloon cuff, and placed in a 50-ml centrifuge tube. The ET tube was suspended in the tube with a syringe needle and was then spun at $220 \mathrm{~g}$ for 30 seconds, yielding 100-300 $\mu 1$ of mucus. Mucus with visible blood contamination was not included in the analysis. Samples were stored at $4^{\circ} \mathrm{C}$ immediately after collection for up to 24 hours for MPT experiments. The data presented here are from 2 male and 3 female subjects (median age of $45 \pm 25$ years in their ages).

Biochemical analysis of CF sputum. For the fluorometric assays of mucin and DNA concentration, aliquots of individual sputum samples were treated with rhDNase at the final concentration of $10 \mu \mathrm{g} / \mathrm{ml}$ and homogenized by vortexing for at least 30 minutes before analysis.

Mucin concentration was determined based on the reaction of 2-cyanoacetamide (Sigma-Aldrich) with 
O-linked glycoproteins (i.e., mucin), as previously described (27). Briefly, the mucin detection solution was prepared by mixing $200 \mu \mathrm{l}$ of $0.6 \mathrm{M}$ 2-cyanoacetamide with $1 \mathrm{ml}$ of $0.15 \mathrm{M} \mathrm{NaOH}$. Fifty microliters of 20 -fold diluted sputum sample was mixed with the detection solution and incubated at $100^{\circ} \mathrm{C}$ for $30 \mathrm{~min}$ utes. The reaction was stopped by an addition of $0.5 \mathrm{ml}$ of $0.6 \mathrm{M}$ borate buffer. Fluorescence intensity was measured at excitation and emission wavelengths of 340 and $420 \mathrm{~nm}$, respectively. A standard curve was generated using known concentrations of mucin from bovine submaxillary gland (Sigma-Aldrich).

DNA concentration was determined based on the reaction between 3,5-diaminobenzoic acid dihydrochloride (DABA; Sigma-Aldrich) and the aldehydes present in DNA. The DNA detection solution consists of $20 \% \mathrm{w} / \mathrm{v}$ DABA in water. Thirty microliters of 5 -fold diluted sputum sample was mixed with $30 \mu \mathrm{l}$ DNA detection solution and incubated at $60^{\circ} \mathrm{C}$ for 1 hour. The reaction was stopped by addition of $1 \mathrm{ml}$ of $1.75 \mathrm{M} \mathrm{HCl}$. Fluorescence intensity was measured at excitation and emission wavelengths of 340 and $420 \mathrm{~nm}$, respectively. A standard curve was generated using known concentrations of DNA from salmon testes (Sigma-Aldrich).

The concentration of mucin disulfide bonds (i.e., cystine) in sputum was measured using a previously described fluorometric assay (23). Briefly, 50- $\mu 1$ sputum aliquots were centrifuged at 21,000 $\mathrm{g}$ for 1.5 hours, and the supernatant was removed. The aliquots were then diluted 10 -fold from the original volume in $8 \mathrm{M}$ guanidine- $\mathrm{HCl}$ (Sigma-Aldrich). In order to ensure that only preexisting disulfide cross-links were reduced, the samples were pretreated with 10\% (v/v) $500 \mathrm{mM}$ iodoacetamide (Sigma-Aldrich) solution and incubated for 1 hour at room temperature. Next, the samples were incubated for 2 hours in 10\% (v/v) 1 M DTT at $37^{\circ} \mathrm{C}$ to reduce all disulfide bridges. To remove excess reagents and soluble factors, each sample was run through 7K MWCO Zeba desalting columns (ThermoFisher Scientific) buffer-exchanged with $50 \mathrm{mM}$ tris$\mathrm{HCl}$ ( $\mathrm{pH}$ 8.0, Sigma-Aldrich). Serial dilutions of $5 \mathrm{mM}$ L-cysteine (Sigma-Aldrich) in $50 \mathrm{mM}$ tris-HCl were used as a standard. Samples and standards were added to a black Maxisorp plate with an equal volume of 2 $\mathrm{mM}$ monobromobimane (Sigma-Aldrich) in $50 \mathrm{mM}$ tris- $\mathrm{HCl}$. The fluorescence was measured at excitation and emission wavelengths of 395 and $490 \mathrm{~nm}$, respectively.

All fluorometric measurements were performed using a microplate reader (BioTek Synergy Mx). Sputum samples were prepared in triplicate, and an average of 3 independent readings was used to obtain mucin, DNA, and cystine concentrations.

The percent solids content of mucus was determined by freeze-drying. Sputum samples were frozen in liquid $\mathrm{N}_{2}$ and placed in a lyophilizer (FreeZone 4.5 Plus; Labconco) for at least 12 hours to completely extract water within individual samples. The ratio of mucus mass before and after the lyophilization is the percent solids content.

Fluorescent dextran staining of CF sputum - sample preparation and microscopy. Confocal microscopy experiments were performed to measure the distribution of $3-\mathrm{kDa}$ Texas red-labeled dextran (Invitrogen), with radius of gyration $R_{\mathrm{g}} \sim 1.5 \mathrm{~nm}$, within CF sputum. Fluorescent dextran has been used in prior studies to measure mucus and PCL thicknesses in primary human airway cultures from CF patients grown at an air-liquid interface (20). Given that it is a neutral, hydrophilic biopolymer, we do not expect dextran to significantly adhere to other biomolecules within the sputum gel and have any effect on sputum microstructure. We also used a very-low-MW dextran $(3 \mathrm{kDa})$ with a small radius of gyration $\left(R_{\mathrm{g}}\right.$ " $\left.1.5 \mathrm{~nm}\right)$ to limit any potential physical entanglements within the sputum gel, with dextran remaining in the fluid phase to stain sputum pore structure.

To prepare samples, 30- $\mu 1$ sputum aliquots were withdrawn using a Wiretrol (Drummond Scientific Company) and added to custom microscopy chambers. Microscopy chambers were prepared using a 1-mm ID Viton o-ring (McMaster Carr) adhered to a glass coverslip with vacuum grease (Dow Corning). From a stock concentration of $10 \mathrm{mg} / \mathrm{ml}$ Texas red-labeled dextran, $0.5 \mu 1$ of stock solution was added to the sputum in the chamber and was mechanically mixed with a pipette tip to distribute dextran throughout the sample. Next, $0.5 \mu 1$ of PS-PEG particles were added to the chamber and were mechanically mixed with a pipette tip to distribute the particles within the sample. The chamber was sealed with a small circular coverslip to prevent evaporation. The samples were equilibrated at room temperature for 1 hour before imaging to allow dissipation of convective flows within the sample due to mixing.

The samples were imaged using a confocal LSM 510 microscope at $\times 63$ magnification with image resolution of $40 \mathrm{~nm} /$ pixel. 3D images of dextran distribution within CF sputum were obtained from 25 $2 \mathrm{D}$ images $(20 \mu \mathrm{m} \times 20 \mu \mathrm{m}$ area) with $0.33 \mu \mathrm{m}$ vertical spacing $(8.25 \mu \mathrm{m}$ total height). Details on image processing are provided in Supplemental Figure 5. The volume of the dextran-filled void space $\left(V_{\mathrm{D}}\right)$ was 
measured using the MATLAB image analysis toolbox and used to determine the porosity, which was calculated as $\phi=V_{\mathrm{D}} / V_{\mathrm{T}}$, where $V_{\mathrm{T}}$ is total volume of the image area $(20 \mu \mathrm{m} \times 20 \mu \mathrm{m} \times 8.25 \mu \mathrm{m})$. MIP diffusion in $2 \mathrm{D}$ was measured within $\mathrm{CF}$ sputum at the center of the same region of interest $(\sim 4 \mu \mathrm{m}$ depth within $3 \mathrm{D}$ imaged area). Videos were recorded at a frame rate of $2.5 \mathrm{~Hz}$ for 100 frames using an EM-CCD camera (Zeiss).

Particle tracking - sample preparation and microscopy. Fluorescent video microscopy experiments were performed to track the motion of nanoparticles in sputum. The samples were processed within 24 hours of collection in order to minimize sample degradation and were not altered in any way prior to experiments in order to retain physiological microstructure. Thirty-microliter sputum aliquots were withdrawn using a Wiretrol (Drummond Scientific Company) and added to custom microscopy chambers. Next, $0.5 \mu 1$ of PS-PEG particles were added to the chamber and were mechanically mixed with a pipette tip to distribute the particles within the sample. By adding this small volume, we can limit any potential dilution effects on sputum microstructure. The chamber was sealed with a small circular coverslip and equilibrated at room temperature for 30 minutes before imaging. Samples were imaged at room temperature using an Axio Observer inverted epifluorescence microscope and $\times 100 / 1.46$ NA oil-immersion objective with image resolution of $25 \mathrm{~nm} /$ pixel (Zeiss). To avoid edge effects due to the presence of glass coverslips, images were taken centrally within the sample approximately $2 \mu \mathrm{m}$ away from the bottom coverslip. Videos were recorded at a frame rate of $15 \mathrm{~Hz}$ for 300 frame, using an EM-CCD camera (Evolve 512; Photometrics). For each sputum sample, 3-5 videos were collected.

MPT analysis. MPT analysis was performed using automated software custom-written in MATLAB (Mathworks), based on a previously developed algorithm (49). The MATLAB software package is described in detail in prior publications $(31,32)$. Briefly, the $x$ and $y$ positions of nanoparticle centers were determined based on an intensity threshold. "Noise" particles also above this brightness threshold showed low circularity and were discarded based on measured eccentricity. Trajectories were then constructed by connecting particle centers between sequential images given an input maximum moving distance between frames. The time-averaged mean squared displacement $[\operatorname{MSD}(\tau)]$ can then be calculated as $\left\langle\Delta r^{2}(\tau)\right\rangle=\langle[x(t$ $+\tau)-x(t)]^{2}+[y(t+\tau)+y(t)]^{2}>$, where $\tau$ is the time lag between frames and angle brackets denote the average over many time points. Median values were determined based on measured MSD for individual MIP and not ensemble averaged over all MIP given the inherent heterogeneity and non-Gaussian distributions of MIP transport in CF sputum (50). We have also found in previous work that MSD measured at $\tau=1$ $s\left(M_{1 s}\right)$ limited the effects of static and dynamic error in MPT experiments $(31,32,51)$. Based on this, median $\log _{10}\left[\mathrm{MSD}_{1 \mathrm{~s}}\right]$ was used as our primary read-out for comparison between individual patient samples.

Particle-tracking microrheology analysis. The viscoelastic properties of hydrogels can be determined using the generalized Stokes-Einstein relation (52), which has been successfully used in prior studies of CF sputum (39), which relates the viscoelastic spectrum $[\mathrm{G}(\mathrm{s})]$ to the Laplace transform of $\left\langle\Delta r^{2}(\tau)\right\rangle,\left\langle\Delta r^{2}(s)\right\rangle$, with the equation $\mathrm{G}(\mathrm{s})=2 k_{\mathrm{B}} T /\left[\pi \mathrm{as}<\Delta \mathrm{r}^{2}(s)>\right]$, where $k_{\mathrm{B}} T$ is thermal energy, $a$ is particle radius, and $s$ is the complex Laplace frequency. By substituting $s$ with $i \omega$ where $i$ is a complex number and $\omega$ is frequency, the complex modulus can then be calculated as $G^{*}(\omega)=G^{\prime}(\omega)+G^{\prime \prime}(i \omega)$. The complex microviscosity, $\eta^{*}(\omega)$, which takes into account both the viscous and elastic moduli, $G^{\prime \prime}$ and $G^{\prime}$ respectively, can be calculated as $\eta^{*}(\omega)=G^{*}(\omega) / \omega$. The pore size of the sputum hydrogel $(\xi)$ can be estimated based on measured $G^{\prime}$ as $\xi \approx$ $\left(k_{\mathrm{B}} T / G^{\prime}\right)^{1 / 3}(53)$. To the limit the effects of static and dynamic error as previously mentioned, MSD at time scales ranging from 0.5 to $5 \mathrm{~s}$ were used in our microrheological analysis. The microviscosity and pore size values were determined from individual MIP trajectories rather than based on ensemble average MSD, given the heterogeneous nature of MIP transport in CF sputum samples.

Statistics. Data were analyzed with parametric statistical tests in SigmaPlot 10.0, including linear regression and 2-tailed Student's $t$ test and a nonparametric Mann Whitney test. Data were considered statistically significant when $P<0.05$. The statistical tests used in each data set are indicated in the figure legends. Power calculations were performed using MATLAB. We determined that 18 patients provided sufficient power $(>95 \%)$ based on the average and sample variance of measured median $\log _{10}\left[\mathrm{MSD}_{1 \mathrm{~s}}\right]$.

Study approval. CF sputum samples were collected after receiving written informed consent and approval from the Johns Hopkins Institutional Review Board (study NA_00046768). Non-CF human airway mucus samples were collected in accordance with a protocol approved by the Johns Hopkins Institutional Review Board (study NA_00038606). Informed consent was not required because we collected a surgical waste product (i.e., ET tubes), and all comorbidity data was deidentified, minimizing potential risk to patients. 


\section{Author contributions}

GAD, JSS, and JH designed experiments. GAD, JJ, and AJ performed experiments. ALT, NEW, and MPB collected patient samples and managed clinical data. GAD, JJ, AJ, ALT, JSS, and JH analyzed the experiments. GAD, NEW, JSS, and JH wrote the manuscript.

\section{Acknowledgments}

This work was supported by the National Institutes of Health (P01HL51811, R01HL127413, and 2P30EY001765) and the Cystic Fibrosis Foundation (HANES07XX0 and HANES15G0).

Address correspondence to: Jung Soo Suk, Center for Nanomedicine at the Wilmer Eye Institute, Johns Hopkins University School of Medicine, 400 N. Broadway, Robert H. and Clarice Smith Building, 6029, Baltimore, Maryland 21231, USA. Phone: 410.614.4526; E-mail: jsuk@jhmi.edu. Or to: Justin Hanes, Center for Nanomedicine at the Wilmer Eye Institute, Johns Hopkins University School of Medicine, 400 N. Broadway, Robert H. and Clarice Smith Building, 6017, Baltimore, Maryland 21231, USA. Phone: 410.614.6513; E-mail: hanes@jhmi.edu.

1. Rowe SM, Miller S, Sorscher EJ. Cystic fibrosis. N Engl J Med. 2005;352(19):1992-2001.

2. Döring G, Flume P, Heijerman H, Elborn JS, Consensus Study Group. Treatment of lung infection in patients with cystic fibrosis: current and future strategies. J Cyst Fibros. 2012;11(6):461-479.

3. Elborn JS, Bell SC. Pulmonary exacerbations in cystic fibrosis and bronchiectasis. Thorax. 2007;62(4):288-290.

4. Gibson RL, Burns JL, Ramsey BW. Pathophysiology and management of pulmonary infections in cystic fibrosis. Am J Respir Crit Care Med. 2003;168(8):918-951.

5. Sanders DB, Bittner RC, Rosenfeld M, Redding GJ, Goss CH. Pulmonary exacerbations are associated with subsequent FEV1 decline in both adults and children with cystic fibrosis. Pediatr Pulmonol. 2011;46(4):393-400.

6. Quinton PM. Cystic fibrosis: impaired bicarbonate secretion and mucoviscidosis. Lancet. 2008;372(9636):415-417.

7. Boucher RC. Airway surface dehydration in cystic fibrosis: pathogenesis and therapy. Annu Rev Med. 2007;58:157-170.

8. Lethem MI, James SL, Marriott C, Burke JF. The origin of DNA associated with mucus glycoproteins in cystic fibrosis sputum. Eur Respir J. 1990;3(1):19-23.

9. Pezzulo AA, et al. Reduced airway surface $\mathrm{pH}$ impairs bacterial killing in the porcine cystic fibrosis lung. Nature. 2012;487(7405):109-113.

10. Hoegger MJ, et al. Impaired mucus detachment disrupts mucociliary transport in a piglet model of cystic fibrosis. Science. 2014;345(6198):818-822.

11. Knowles MR, Boucher RC. Mucus clearance as a primary innate defense mechanism for mammalian airways. J Clin Invest. 2002;109(5):571-577.

12. Rubin BK. Physiology of airway mucus clearance. Respir Care. 2002;47(7):761-768

13. Rubin BK. Mucus, phlegm, and sputum in cystic fibrosis. Respir Care. 2009;54(6):726-32; discussion 732.

14. Fahy JV, Dickey BF. Airway mucus function and dysfunction. N Engl J Med. 2010;363(23):2233-2247.

15. Randell SH, Boucher RC, University of North Carolina Virtual Lung Group. Effective mucus clearance is essential for respiratory health. Am J Respir Cell Mol Biol. 2006;35(1):20-28.

16. Elizur A, Cannon CL, Ferkol TW. Airway inflammation in cystic fibrosis. Chest. 2008;133(2):489-495.

17. Cohn L. Mucus in chronic airway diseases: sorting out the sticky details. J Clin Invest. 2006;116(2):306-308.

18. Serisier DJ, Carroll MP, Shute JK, Young SA. Macrorheology of cystic fibrosis, chronic obstructive pulmonary disease \& normal sputum. Respir Res. 2009;10:63.

19. Robinson M, Bye PT. Mucociliary clearance in cystic fibrosis. Pediatr Pulmonol. 2002;33(4):293-306.

20. Button B, et al. A periciliary brush promotes the lung health by separating the mucus layer from airway epithelia. Science. 2012;337(6097):937-941.

21. Hill DB, et al. A biophysical basis for mucus solids concentration as a candidate biomarker for airways disease. PLoS One. 2014;9(2):e87681.

22. Anderson WH, et al. The relationship of mucus concentration (hydration) to mucus osmotic pressure and transport in chronic bronchitis. Am J Respir Crit Care Med. 2015;192(2):182-190.

23. Yuan S, et al. Oxidation increases mucin polymer cross-links to stiffen airway mucus gels. Sci Transl Med. 2015;7(276):276ra27.

24. Matsui H, et al. Reduced three-dimensional motility in dehydrated airway mucus prevents neutrophil capture and killing bacteria on airway epithelial surfaces. J Immunol. 2005;175(2):1090-1099.

25. Matsui $\mathrm{H}$, et al. A physical linkage between cystic fibrosis airway surface dehydration and Pseudomonas aeruginosa biofilms. Proc Natl Acad Sci U S A. 2006;103(48):18131-18136.

26. Suk JS, et al. The penetration of fresh undiluted sputum expectorated by cystic fibrosis patients by non-adhesive polymer nanoparticles. Biomaterials. 2009;30(13):2591-2597.

27. Schuster BS, Suk JS, Woodworth GF, Hanes J. Nanoparticle diffusion in respiratory mucus from humans without lung disease. Biomaterials. 2013;34(13):3439-3446.

28. Lai SK, et al. Rapid transport of large polymeric nanoparticles in fresh undiluted human mucus. Proc Natl Acad Sci U S A. 2007;104(5):1482-1487.

29. Lai SK, Wang YY, Hida K, Cone R, Hanes J. Nanoparticles reveal that human cervicovaginal mucus is riddled with pores 
larger than viruses. Proc Natl Acad Sci U S A. 2010;107(2):598-603.

30. Lai SK, et al. Drug carrier nanoparticles that penetrate human chronic rhinosinusitis mucus. Biomaterials. 2011;32(26):6285-6290.

31. Schuster BS, et al. Overcoming the cystic fibrosis sputum barrier to leading adeno-associated virus gene therapy vectors. Mol Ther. 2014;22(8):1484-1493.

32. Schuster BS, Ensign LM, Allan DB, Suk JS, Hanes J. Particle tracking in drug and gene delivery research: State-of-the-art applications and methods. Adv Drug Deliv Rev. 2015;91:70-91.

33. App EM, et al. Sputum rheology changes in cystic fibrosis lung disease following two different types of physiotherapy: flutter vs autogenic drainage. Chest. 1998;114(1):171-177.

34. Innes AL, et al. Ex vivo sputum analysis reveals impairment of protease-dependent mucus degradation by plasma proteins in acute asthma. Am J Respir Crit Care Med. 2009;180(3):203-210.

35. Suk JS, Lai SK, Boylan NJ, Dawson MR, Boyle MP, Hanes J. Rapid transport of muco-inert nanoparticles in cystic fibrosis sputum treated with $\mathrm{N}$-acetyl cysteine. Nanomedicine (Lond). 2011;6(2):365-375.

36. Davies JC, Alton EW. Monitoring respiratory disease severity in cystic fibrosis. Respir Care. 2009;54(5):606-617.

37. Mogayzel PJ, et al. Cystic fibrosis pulmonary guidelines. Chronic medications for maintenance of lung health. Am J Respir Crit Care Med. 2013;187(7):680-689.

38. Mrsny RJ, Daugherty AL, Short SM, Widmer R, Siegel MW, Keller GA. Distribution of DNA and alginate in purulent cystic fibrosis sputum: implications to pulmonary targeting strategies. J Drug Target. 1996;4(4):233-243.

39. Dawson M, Wirtz D, Hanes J. Enhanced viscoelasticity of human cystic fibrotic sputum correlates with increasing microheterogeneity in particle transport. J Biol Chem. 2003;278(50):50393-50401.

40. Cone RA. Barrier properties of mucus. Adv Drug Deliv Rev. 2009;61(2):75-85.

41. Ensign LM, Schneider C, Suk JS, Cone R, Hanes J. Mucus penetrating nanoparticles: biophysical tool and method of drug and gene delivery. Adv Mater Weinheim. 2012;24(28):3887-3894.

42. Henderson AG, et al. Cystic fibrosis airway secretions exhibit mucin hyperconcentration and increased osmotic pressure. J Clin Invest. 2014;124(7):3047-3060.

43. Sanders NN, De Smedt SC, Van Rompaey E, Simoens P, De Baets F, Demeester J. Cystic fibrosis sputum: a barrier to the transport of nanospheres. Am J Respir Crit Care Med. 2000;162(5):1905-1911.

44. Suk JS, et al. N-acetylcysteine enhances cystic fibrosis sputum penetration and airway gene transfer by highly compacted DNA nanoparticles. Mol Ther. 2011;19(11):1981-1989.

45. Rubin BK. Surface properties of respiratory secretions: relationship to mucus transport. In: Baum G, ed. Cilia, Mucus, and Mucociliary Interactions. New York: Marcel Dekker; 1998: 317-324.

46. Teng D-y, et al. Synthesis and characterization of in situ cross-linked hydrogel based on self-assembly of thiol-modified chitosan with PEG diacrylate using Michael type addition. Polymer. 2010;51(3):639-646.

47. Zheng Shu X, Liu Y, Palumbo FS, Luo Y, Prestwich GD. In situ crosslinkable hyaluronan hydrogels for tissue engineering. Biomaterials. 2004;25(7-8):1339-1348.

48. Burdick JA, Prestwich GD. Hyaluronic acid hydrogels for biomedical applications. Adv Mater Weinheim. 2011;23(12):H41-H56.

49. Crocker J, Grier D. Methods of Digital Video Microscopy for Colloidal Studies. J Colloid Interface Sci. 1996;179(2):298-310.

50. Feinstein AR. Principles of medical statistics. Boca Raton, Florida: Chapman and Hall/CRC; 2001.

51. Kim AJ, et al. Use of single-site-functionalized PEG dendrons to prepare gene vectors that penetrate human mucus barriers. Angew Chem Int Ed Engl. 2013;52(14):3985-3988.

52. Mason TG, Weitz DA. Optical measurements of frequency-dependent linear viscoelastic moduli of complex fluids. Phys Rev Lett. 1995;74(7):1250-1253.

53. De Gennes PG. Scaling concepts in polymer physics. Ithaca, New York: Cornell University Press; 1979 